\section{THE TOOLS OF AUTOMATED GENERALIZATION AND BUILDING GENERALIZATION IN AN ARCGIS ENVIRONMENT}

\section{ABSTRACT}

Cartographic generalization plays an important role in the creation of maps. The implementation of generalization rules in the form of generalization tools in a GIS environment is an important part of digital cartography today. The article describes possibilities for the use of vector dataset generalization tools in an ArcGIS 9.2 environment. The main goal of the paper is to design and verify a generalization model of buildings for cartographic output on a scale of $1: 10000$ on the basis of BD GIS. The limit values were defined, and two approaches to building generalization on a scale of $1: 10,000$ were defined and tested (Generalization A, Generalization B).

\section{Veronika DROPPOVA}

mail: veronika droppova@gmail.com

Research field: Geographic Information System, Modelling, Cartographic Generalization, automated, Basic Database for Geographic Information System (BD GIS), Generalization of Buildings

Department of Mapping and Land Consolidation Faculty of Civil Engineering, Slovak University of Technology in Bratislava, Radlinského 11 81368 Bratislava

\section{KEY WORDS}

- geographic Data,

- geographic Information System,

- BD GIS,

- cartographic Generalization,

- generalization of Buildings.

\section{INTRODUCTION}

Actual computer technologies and practices are a positive proposition for the creation of maps. They effectively assist in the implementation of traditional and also new cartographic methods in a computer environment. A Geographic Information System (GIS) is a useful tool for modeling geographical spaces and is still increasing in its importance and position in society.

The creation of computer maps has a number of advantages in comparisson with classical cartographic methods [5]:

- the creation, production and printing of maps is quicker and more effective,

- the selection and possibility of cartographic projections and conversion of scale factors,

- flexibility in assembling map contents,
- possibility of obtaining, relaying and completing data for the creation of maps,

- large range of possibilities for visual techniques.

\section{PROBLEMS WITH GENERALIZATION}

Often there is a situation when it is not possible to strictly and geometrically express images of objects, relations or processes. A simplified image of a feature is created by leaving out irrelevant details. The scale of a map is not the only factor which affects generalization. The purpose of the map is another factor. The purpose determines what is important, which objects or categories of objects to use, and the symbology of objects. Each generalization process is a combination of several operations; the procedure is not unified, and the result is a specific compromise. 
The achievement of automated generalization is represented by the knowledge and skills obtained through manual generalization of the explicit, and logical rules in a computer environment.

ArcGIS contains a universal and efficient system of tools, which are grouped in toolboxes and toolsets. The tools are summoned by a dialog box and command line; it is also possible to combine them by scripting languages (e.g. Python) or using ModelBuilder (a component of ArcGIS). ArcGIS contains a system of tools for generalizating rasters as well as vector datasets [1].

\subsection{Generalization of a Raster Dataset in ArcGIS}

Sometimes a raster dataset contains data that is erroneous or irrelevant to the analysis or is too detailed. For instance, if a raster dataset is a satellite image, it may contain many small and isolated areas that are misclassified. The generalization functions assist in identifying such areas and automating the assignment of more reliable values for the cells that make up the areas.

The generalization tools of a raster dataset are [1]:

- Aggregate - generates a reduced resolution version of a raster,

- Boundary Clean - smoothes the boundaries between zones by expanding and shrinking them,

- Expand - expands particular zones of a raster by a specified number of cells,

- Majority filter - replaces cells in a raster based on the majority of their contiguous neighboring cells,

- Nibble - replaces the cells of a raster corresponding to a mask with the values of the nearest neighbors,

- Region group - records the identity of the connected region to which it belongs; for each cell in the output a unique number is assigned to each region,

- Shrink - shrinks the selected zones by a specified number of cells,

- Thin - thins rasterize linear features by reducing the number of cells representing the width of the features.

The generalization tools work in zones (Region group, Nibble, Shrink, Thin, Expand), on zone edges (Boundary Clean, Majority Filter) and alter the resolution of the data (Aggregate). Any two or more cells with the same value belong to the same zone. A zone can consist of cells that are adjacent, disconnected, or both.

\subsection{Generalization Of a Vector Dataset in ArcGIS}

The tools in the Generalization toolset make data less detailed and less complex for analysis and representation in reduced scales or for other special purposes. The tools simplify data while maintaining their geographic characteristics and integrity. For example, rivers are simplified by removing their small fluctuations without destroying their essential shapes. Although generalization is a complicated and subjective task, and the rules are not well defined, generalization tools automate the process and meet the common generalization requirements as much as possible [2, 4].

Generalization tools for a vector dataset [1]:

- Aggregate Polygons,

- Collapse Dual Lines To Centerline,

- Dissolve,

- Eliminate,

- Simplify Building,

- Simplify Line,

- Simplify Polygon,

- Smooth Line.

The next part of the paper is focused on cartographic generalization, testing and verification of generalization tool possibilities for vector datasets in the ArcGIS 9.2 on the basis of BD GIS in the model locality of Senec.

\section{Aggregate Polygons}

Aggregate Polygons combine polygons within a specified distance of each other into new polygons.

It is necessary to define the [1]:

- Aggregation Distance - the distance between polygon boundaries for aggregation must be greater than zero,

- Minimum Area - the minimum area for an aggregated polygon to be retained; the default value is zero, i.e., to keep all the polygons,

- Minimum Hole Size - the minimum size of a polygon hole to be retained; the default value is zero, i.e., to keep all the polygon holes.

Depend on the characteristic shape of the input features, it is possible to aggregate orthogonal (buildings, blocks) or nonorthgonal polygons (natural features, such as vegetation or soil polygons, Fig. 1).

\section{Collapse Dual Lines To Centerline}

Derives centerlines from dual-line (or double-line) features, such as roads, based on specified width tolerances (Fig. 2). It is a tool for a road network generalization, but it only solves simple crossing.

\section{Dissolve}

Aggregates features based on specified attributes (Fig. 3), it can be statistically data of object, area, perimeter. It is tool for generalization of areal objects (e.g. forest area, ground cover). 


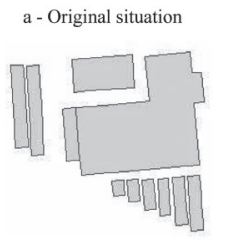

b - Aggregate Polygons - orthogonal
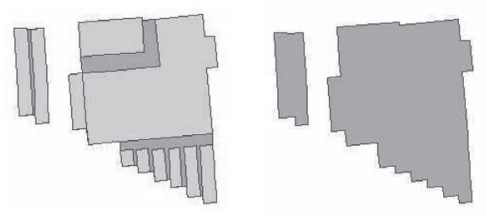

c- Aggregate Polygons - non-orthogonal
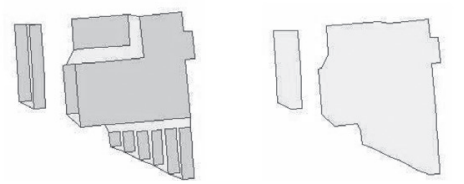

Fig. 1 The use of the Aggregate Polygons tool.
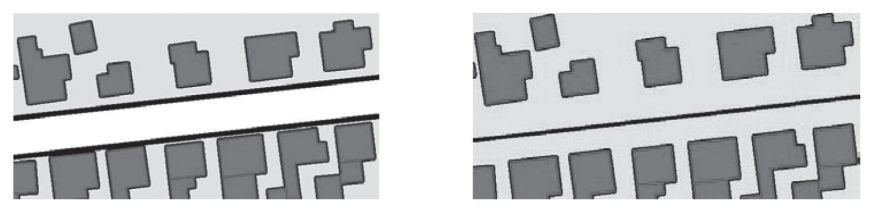

Fig. 2 The use of the Collapse Dual Lines To Centerline.

a - Original situation

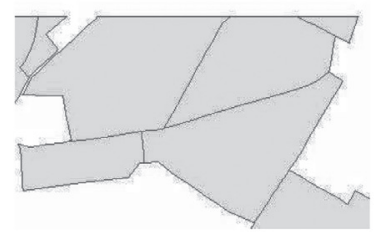

Fig. 3 The use of the Dissolve tool.

\section{Eliminate}

Merges the selected polygons with neighboring polygons with the largest shared border or the largest area. This tool is useful for area generalization, e.g. small area, which is too small for display in reduced scale, it is better assignment it to adjacent area (Fig. 4).

\section{Simplify Building}

Simplifies the boundary or footprint of building polygons while maintaining their essential shape and size (Fig. 5).

Defined parameters [1]:

- Simplify tolerance - sets the tolerance for building simplification, a tolerance must be specified and it must be greater than zero,

- Minimum area - sets the minimum area for a simplified building a - undefined soil (white) and lake

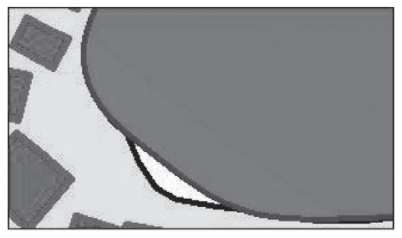

Fig. 4 The use of the Eliminate tool.
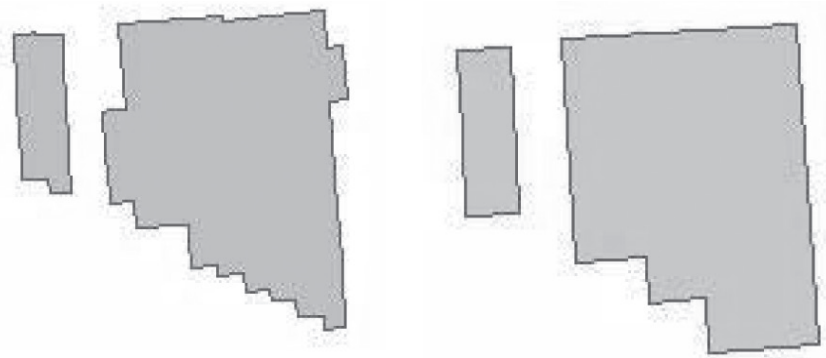

Fig. 5 Buildings before and after using the Simplify Building tool.

to be retained in feature units; the default value is zero, that is, to keep all the buildings.

This tool is applied to a whole object, not only for an object segment. The tool simplifies separate buildings and buildings connected with straight lines (as a group). It does not work with buildings connected in complicated ways.

\section{Simplify Line}

Simplifies a line by removing small fluctuations or extraneous bends from it while preserving its essential shape (Fig. 6). It is a tool used for the generalization of networks of roads and water.

Defined parameters [1]:

- Algorithm:

- Point Remove - keeps the so-called critical points that depict the essential shape of a line and removes all other points,

- Bend Simplify - keeps the main shape of a line and removes extraneous bends,

- Maximum Allowable Offset - the tolerance that determines the degree of simplification; it must be specified and it must be greater than zero:

- for Point Remove - the tolerance is the maximum allowable offset,

- for Bend Simplify - the tolerance is the length of the reference bend baseline. 


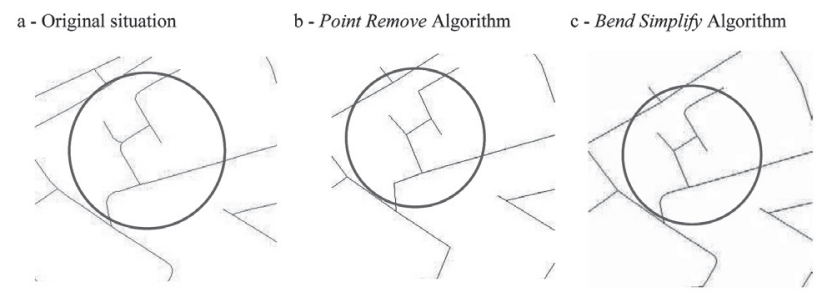

Fig. 6 The simplification of a road network using the Simplify Line tool.
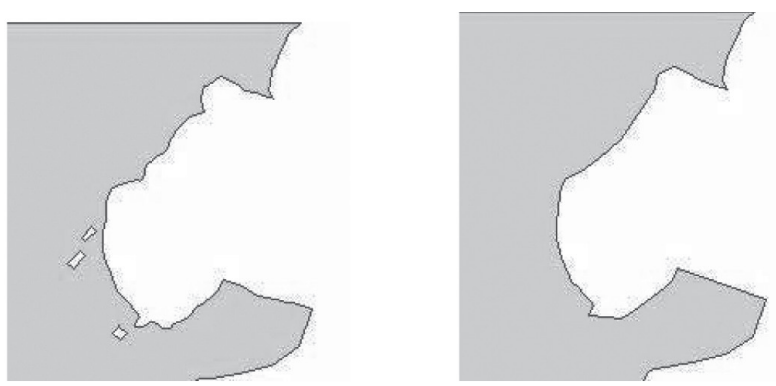

Fig. 7 Polygon before and after using Simplify Polygon tool.

\section{Simplify Polygon}

Simplifies a polygon by removing small fluctuations or exraneous bends from its boundary, while preserving its essential shape (Fig. 7). This tool is used for polygon generalization on reduced scales.

It has to be defined [1]:

- Algorithm as well as Simplify Line

- Maximum Allowable Offset

- Minimum Area - sets the minimum area for a simplified polygon to be retained; the default value is zero, that is, to keep all the polygons.

\section{Smooth Line}

Smoothes a line to improve its aesthetic or cartographic quality. The use of this tool is in Fig 8.

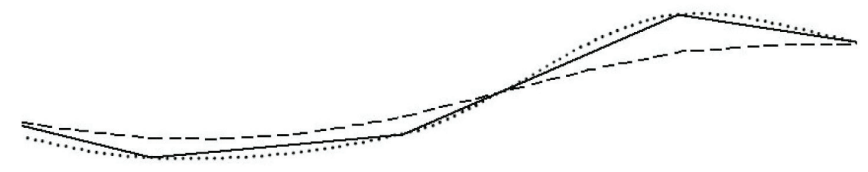

Fig. 8 The use of the Smooth Line tool-original line-solid line, after the dash-and-dot line Algorithm Peak and the dotted line Bezier Interpolation Algorithm.
It has to be defined [1]:

- Algorithm:

- Peak - stands for Polynomial Approximation with Exponential Kernel; it calculates a smoothed line that will not pass through the input line vertices,

- Bezier Interpolation - fits Bezier curves between vertices; the resulting line passes through the vertices of the input line,

- Smoothing Tolerance - sets a tolerance used by the Peak algorithm; it must be specified, and it must be greater that zero.

\section{PROPOSED BUILDING COMPONENT GENERALIZATION FOR A BASIC MAP ON A SCALE OF $1: 10,000$}

The principle of automated generalization is in the settings of the generalization limits, the object of which has to be met in order to be displayed on a given scale. If the object does not fit those limits, it is changed or cancelled.

The generalization options available in an ArcGIS environment were tested on the BD GIS by constructing a generalized cartographic output of the Slovak Republic Basic Map on a scale of $1: 10,000$. The town of Senec and its surroundings $\left(13 \mathrm{~km}^{2}\right)$ were selected as the locality for the model.

Generalization tools can be applied to an entire feature class or only for selected objects and can also be combined to achieve the results needed. Two generalization tools are usually used for constructing a generalization - Simplify Building and Aggregate Polygons.

According to Fig. 9, it is clear that for constructing a generalization, the following has to be defined [3]:

- The minimal edge of an object,

- The minimal distance between two objects,

- The minimal area of an interior polygon,

- The minimal area of an object.

The main purpose was testing the Simplify Building and Aggregate Polygons generalization tools on the model locality of Senec and comparing the results.

Two approaches were tested:

- Generalization A:

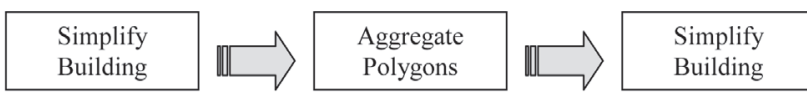

- Generalization B:

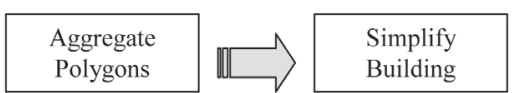




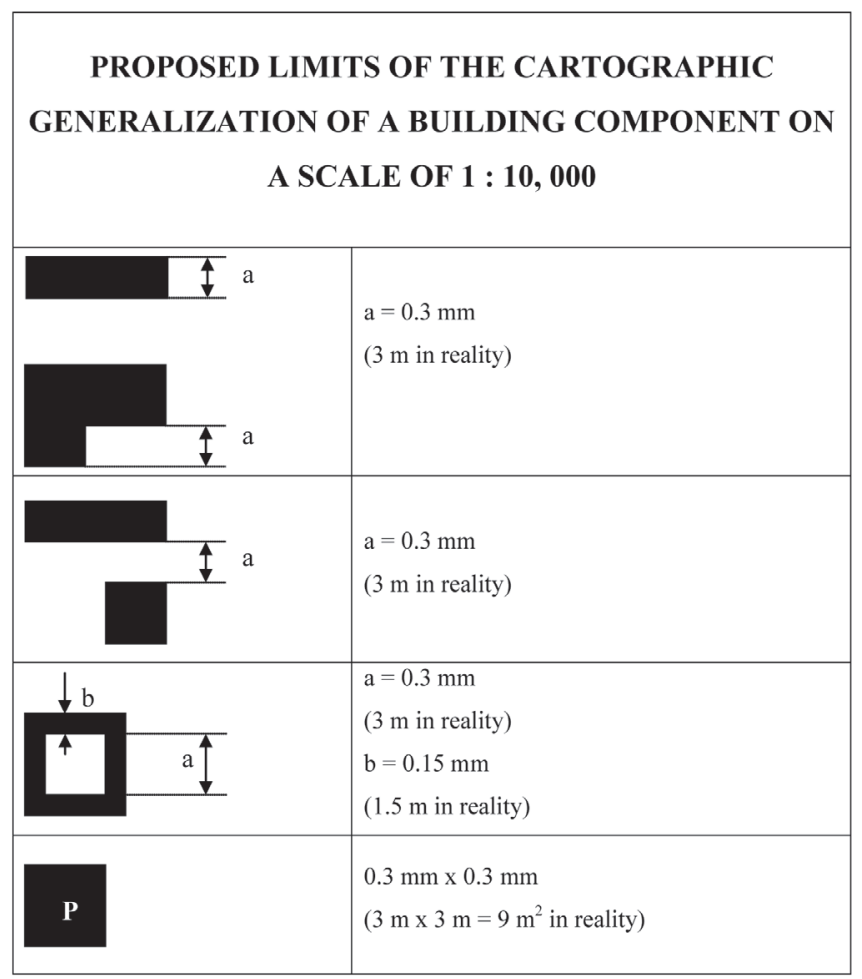

Fig 9 Proposed limits of the cartographic generalization of a building component on a scale of $1: 10,000$ [3].

Three localities were selected in the model locality. Each locality had an area of $1000 \mathrm{~m} \times 1000 \mathrm{~m}$, but with a different density of the built-up area. Both approaches with limits (Tab. 1, 2) were applied in the localities according to Fig. 9.
The indicator for the assessment of the generalization was the number of objects, the areas of the buildings (before and after the generalization) and the graphic results. A numerical representation of the generalized localities is in Tabs. 3 and 4. There are also the results of the generalization phases, but only the resulting values were compared.

The difference between the generalization approaches is minor. Generalization A is one step slower, but the numerical and graphic results prove that it is superior. Generalization A reduces the number of buildings less than generalization B (even though the difference is not major), and the area of the buildings is lower than B.

The graphic outputs of the generalization approaches differ only in the details (the arrangement of the aggregate buildings, the remaining or cancelling of the wall of a niche, etc.). These differences come from the generalization sequences (i.e., the application of the particular tools). The results of approach A are not exactly perfect, but there are fewer such situations in comparison with approach B. So later there will be an advancement during manual editing.

Picture 10 shows some situations where approach A was better than B. Approach A is more time consuming, which is its main disadvantage. The time aspect of this generalization process and the problematic areas are the main disadvantages in creating the cartographic output. These problems can be handled by creating generalization models using ModelBuilder - an application of ArcGIS (for more information, see [3]).

\section{CONCLUSIONS}

There is no universal procedure for solving generalization problems. The process depends on the author's skills and practice, but also

Tab. 1 Definition of the limits of the tools of Generalization A.

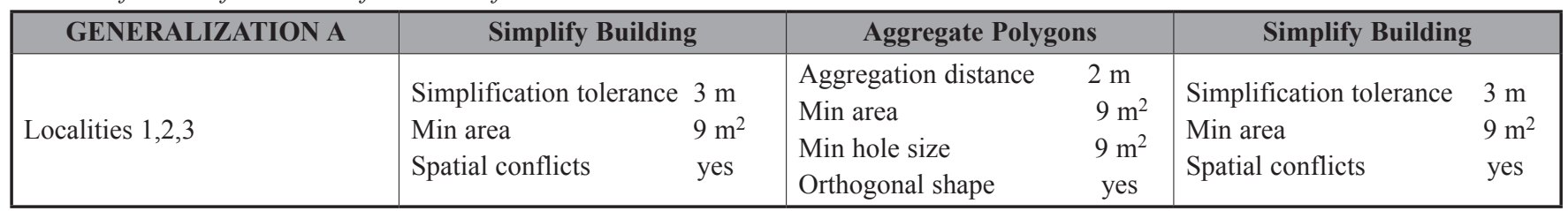

Tab. 2 Definition of the limits of the tools of Generalization B.

\begin{tabular}{|c|ll|ll|}
\hline \multicolumn{1}{|c|}{ GENERALIZATION B } & \multicolumn{2}{c|}{ Aggregate Polygons } & \multicolumn{2}{c|}{ Simplify Building } \\
\hline \multirow{4}{*}{ Locality $1,2,3$} & Aggregation distance & $2 \mathrm{~m}$ & Simplification tolerance & $3 \mathrm{~m}$ \\
& Min area & $9 \mathrm{~m}^{2}$ & Min area & $9 \mathrm{~m}^{2}$ \\
& Min hole size & $9 \mathrm{~m}^{2}$ & Spatial conflicts & yes \\
& Orthogonal shape & yes & & \\
\hline
\end{tabular}


2011/1 PAGES $1-7$

Tab. 3 Numerical representation of Generalization A.

\begin{tabular}{|c|l|c|c|c|c|}
\hline \multicolumn{2}{|c|}{ GENERALIZATION A } & Basis & Simplify Building & Aggregate Polygons & Simplify Building \\
\hline \multirow{2}{*}{ Locality 1} & Number of buildings & 1211 & 1209 & 630 & 630 \\
\cline { 2 - 6 } & Total building areas $\left[\mathrm{m}^{2}\right]$ & 171,770 & 172,050 & 172,591 & 172,766 \\
\hline \multirow{2}{*}{ Locality 2 } & Number of buildings & 407 & 407 & 253 & 253 \\
\cline { 2 - 6 } & Total building areas $\left[\mathrm{m}^{2}\right]$ & 82,169 & 82,218 & 82,334 & 82,479 \\
\hline \multirow{2}{*}{ Locality 3 } & Number of buildings & 649 & 649 & 329 & 329 \\
\cline { 2 - 6 } & Total building areas $\left[\mathrm{m}^{2}\right]$ & 79,862 & 79,966 & 80,479 & 80,610 \\
\hline
\end{tabular}

Tab. 4 Numerical representation of Generalization B.

\begin{tabular}{|c|l|c|c|c|}
\hline \multicolumn{2}{|c|}{ GENERALIZATION B } & Basis & Aggregate Polygons & Simplify Building \\
\hline \multirow{2}{*}{ Locality 1 } & Number of buildings & 1211 & 629 & 629 \\
\cline { 2 - 5 } & Total building areas $\left[\mathrm{m}^{2}\right]$ & 171,770 & 172,387 & 172,889 \\
\hline \multirow{2}{*}{ Locality 2} & Number of buildings & 407 & 251 & 251 \\
\cline { 2 - 5 } & Total building areas $\left[\mathrm{m}^{2}\right]$ & 82,169 & 82,351 & 82,626 \\
\hline \multirow{2}{*}{ Locality 3 } & Number of buildings & 649 & 328 & 328 \\
\cline { 2 - 5 } & Total building areas $\left[\mathrm{m}^{2}\right]$ & 79,862 & 80,479 & 80,649 \\
\hline
\end{tabular}

a - Situation 1 - Original b-Situation 1 - Generalization A c - Situation 1 - Generalization B
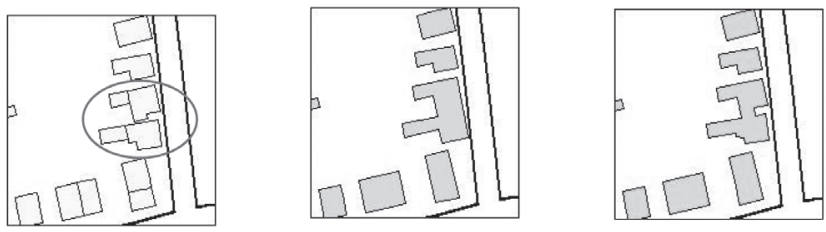

d - Situation 2 - Original

e - Situation 2 - Generalization A f - Situation 2 - Generalization B
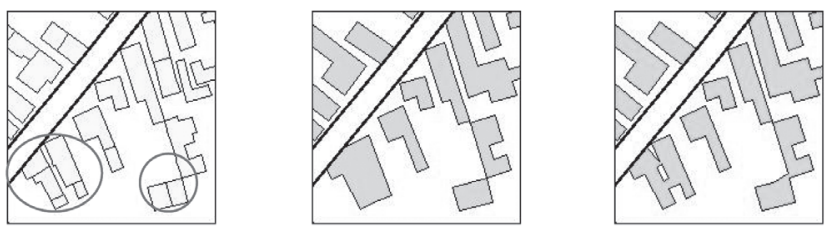

g - Situation 3 - Original

h-Situation 3 - Generalization A i - Situation 3 - Generalization B
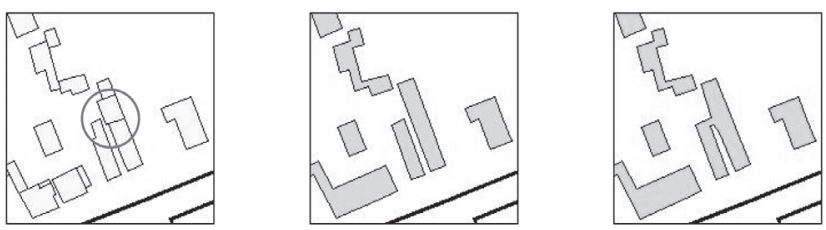

on the specific situations which must be solved. The generalization tools included in the GIS software can accelerate the generalization process, and good results can be achieved.

The ArcGIS environment has a set of generalization tools for rasters and also for vector datasets. The generalization tools for vector datasets were tested in the model locality of Senec. The proposed building generalization for the basic map on a scale of $1: 10,000$ on BD GIS was processed using a combination of two generalization tools - Simplify Building and Aggregate Polygons. The two generalization approaches (Generalization A, Generalization B) were proposed and tested by the generalization limits.

The generalization results were rated by the number of objects, the areas of the buildings (before and after the generalization) and the graphic results. The difference between the approaches is not significant, but Generalization A is better. It reduced the number of buildings less than B did, and also the area of the buildings is lower than with $\mathrm{B}$. The disadvantage of approach $\mathrm{A}$ is the delay from using one more tool application. This problem can be handled by creating a generalization model. Automated generalization models for proposed approaches could be created using ModelBuilder (an ArcGIS application).

Quite a few authors have dealt with generalization problems. Cartographic generalization issues include a lot of open problems, so there is a lot of room for solving them.

Fig. 10 Comparative advantages of Generalization A. 


\section{IDOJLS JOUSSILL \\ 0."

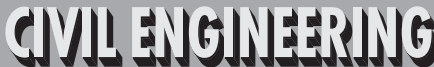

2011/1 PAGES $1-7$

\section{REFERENCES}

[1] ArcGIS 9.2 ESRI, Desktop Help, Desktop Help Online http://webhelp.esri.com/arcgisdesktop/9.2/index. cfm?TopicName=welcome.

[2] ESRI (2000): Map Generalization in GIS: Practical Solutions with Workstation ArcInfo Software, 20pp. http://downloads. esri.com/support/whitepapers/ao_/?ap_Generalization.pdf, (2007-02-10).

[3] DROPPOVÁ, V (2010): Kartografické modelovanie v prostredí GIS (Cartographic Modelling in a GIS Environment). Dissertation Thesis, Slovak University of Technology in Bratislava, Faculty of Civil Engineering; Department of Mapping and Land Consolidation, 165 pp, (in Slovak).
[4] DROPPOVÁ, V., FENCÍK, R. (2007): Možnosti nástrojov automatizovanej generalizácie v prostredí ArcGIS (Possibilities of Automatized Generalization Tools in an ArcGIS Environment). In: Geodetic and Cartographic Review, Prague: Vesmír, spol. s r. o., vol. 53/95, No. 7 - 8, pp. 156 161, ISSN 0016-7096, (in Slovak).

[5] PRAVDA, J., KUSENDOVÁ, D (2004): Počítačová tvorba tematických máp (Computer Creation of Thematic Maps). Comenius University in Bratislava, 264pp., ISBN 80-2232011-0 (in Slovak). 